\title{
Modeling and computation of heterogeneous implicit solvent and its applications for biomolecules
}

\begin{abstract}
Description of inhomogeneous dielectric properties of a solvent in the vicinity of ions has been attracting research interests in mathematical modeling for many years. From many experimental results, it has been concluded that the dielectric response of a solvent linearly depends on the ionic strength within a certain range. Based on this assumption, a new implicit solvent model is proposed in the form of total free energy functional and a quasi-linear Poisson-Boltzmann equation (QPBE) is derived. Classical Newton's iteration can be used to solve the QPBE numerically but the corresponding Jacobian matrix is complicated due to the quasi-linear term. In the current work, a systematic formulation of the Jacobian matrix is derived. As an alternative option, an algorithm mixing the Newton's iteration and the fixed point method is proposed to avoid the complicated Jacobian matrix, and it is a more general algorithm for equation with discontinuous coefficients. Computational efficiency and accuracy for these two methods are investigated based on a set of equation parameters. At last, the QPBE with singular charge source and piece-wisely defined dielectric functions has been applied to analyze electrostatics of macro biomolecules in a complicated solvent. A set of computational algorithms such as interface method, singular charge removal technique and the Newtonfixed-point iteration are employed to solve the QPBE. Biological applications of the proposed model and algorithms are provided, including calculation of electrostatic solvation free energy of proteins, investigation of physical properties of channel pore of an ion channel, and electrostatics analysis for the segment of a DNA strand.
\end{abstract}

Keywords: Implicit solvent model; Heterogeneous solvent; Nonlinear iterative methods; Electrostatic potential

DOI 10.2478/mlbmb-2014-0008

Received September 9, 2014; accepted November 3, 2014

\section{Introduction}

Ionic solvent is extremely important to almost all biological processes involving biomolecules under physiological conditions, such as protein-folding, protein-ligand binding, signal transduction, or DNA specification and transcription, because water comprises $65-90 \%$ of cellular mass and multiple ion species are dissolved. It is a key step and prerequisite to study the energetics of interactions between solute molecules and ionic solvent in order to provide quantitative description and analysis in understanding these phenomena. However, resolving the interactions of ions in aqueous solution, or modeling and simulation of ionic solvent itself could be an intimidating task, due to the intense interactions among ionic species, water molecules, and macrobiomolecules. For example, in a non-dilute solvent, ions are subject to strong charge correlations and fluctuations via Coulomb's force, which forms a typical many-body problem. These long-range and shortrange interactions among the particles in a solvent are summarized as the ion-specific hydration effects.

^Corresponding Author: Duan Chen: Department of Mathematics and Statistics, University of North Carolina at Charlotte, Charlotte, NC, 28223, USA, E-mail:dchen10@uncc.edu 
Molecular dynamics or quantum mechanics seem to be the natural choices to capture a detailed microscopic picture of these interactions. But for a system consisting of millions of charged particles, simulations are still prohibitively expensive even with current rapidly growing computational powers. Alternatively, the Poisson-Boltzmann (PB) theory is a mean-filed approach which accounts for the electrostatic interactions in electrolyte solution implicitly: water molecules are modeled as structureless dielectric continuum and mobile ions are described in terms of ionic concentrations. Boltzmann distribution of ion concentration is obtained from the variation of Helmholtz free energy of the system in equilibrium states, and provides impressive results in various applications such as calculations of solvation free energies or $p K_{a}$ values of proteins. Computationally, a vast variety of techniques for numerically solving the PBE, including finite difference methods (FDMs)[1-4], finite element methods (FEMs) [5], and boundary integral methods (BIMs) [6, 7], have been developed in the past few decades and been incorporated into popular molecular simulation software packages, such as DelPhi [8], ZAP [9], UHBD [10], MEAD [11], APBS [12], AMBER [2, 13] and CHARMM [14, 15]. Recently, highly accurate and efficient numerical schemes of PBE and their applications in molecular biology have attracted much interests in the community of computational mathematics. Novel algorithms, such as FDM based matched interface and boundary (MIB) method[16-18], immersed interface method (IIM) [19, 20], BIM based treecode acceleration technique[21], are developed to handled solute-solvent interface and singular charges of proteins appearing in the PBE.

Nevertheless, the PB theory on the mean-field level admits well-understood drawbacks and limitations. Since ions are considered as point-like particles independent to each other in the continuum approach, all the interactions among the particles in the solvent, including finite size effects of ions, ion-ion charge correlation and fluctuation, or polarizability of ions on solvent and themselves, are not included[22, 23]. Another major issue of the PB theory is over-simplifying water as a structureless continuum with a uniform dielectric constant, while water molecules are dipoles which response to local electrical filed and they are polarizable by individual ions. Neglecting these ion-ion and ion-water interactions, the PB theory is more successful in case of low ionic strength or far away from the charged dielectric boundary. It is problematic or even entirely fails to desirable ionic distribution and electrostatic near strongly charged boundary or non-dilute solvent. Much efforts have been devoted to extend the PB theory in order to address the above-mentioned issues. To name a few, a lattic-gas entropy of ions in a mean-filed form was systematically derived via a field-theory approach to take into account the steric effects of hydration shells[24]. In [25], a phenomenological mean-field free-energy has been proposed with dielectric response of solvent depending linearly on ionic concentrations. A LandauGinzburg-like free energy functional was developed in [26] for the over-screening and crowding of ions near charged surfaces. More related work can be found in the recent review[23]. In[27], density functional theory was used to model the charged hard sphere fluid. All the resulting modified PB models are subject to strong nonlinearity of the partial differential equations, thus it has attracted much interests in mathematical analysis and scientific computations. For example, ion size induced repulsion effects have been analyzed in [28, 29] and numerical computations of for some heterogeneous solvent models are presented in [30-32].

The objective of the current work is to propose a new mean-filed model and develop corresponding computational algorithms, targeting at the heterogeneous dielectric response of an implicit solvent. First of all, the model is built on the framework of total free energy functional. In the past decades, a series of successful mathematical models have been developed on top of the free energy functional, for multi-physics phenomena[33], differential geometry based multi-scale treatment[34], complex fluid[35], classic or quantum mechanical modeling for dynamics of charged particles [36-39]. This formulation gives a clear picture of physical components and their mutual interactions in a system and it is also a powerful tool to perform necessary mathematical analysis[40, 41]. Secondly, this model is based on direct experimental observations in stead of phenomenological assumptions. Physically, the orientation of dipolar moments and the arrangement of hydrogen-bond networks of water molecules in the vicinity of an ion are restricted and different from those out of the ion-hydration shell. Hence, the static dielectric function of an electrolyte solution is generally lower than that of the pure water, because of ionic polarizability and formation of ion hydration shells. Recent experiments[42-44] and molecular dynamic simulations [45] found that this dielectric decrement linearly depends on the concentration of specific ionic species in the physiological conditions. Therefore, with the simple assumption that the dielectric function in the total energy linearly depends on the local ionic strength, 
a modified Boltzmann distribution with a dielectric decrement factor is derived for a system in equilibrium state and we eventually obtain a quasi-linear PB equation (QPBE).

Efficient computational algorithms are developed for the resulting governing equation aiming at its nonlinearity. For the conventional PBE, the Newton's method, or its extensions, (damped) inexact Newton's method are applied to handle the semi-linear term [46]. Recently, an operator splitting ADI scheme was proposed to solve the pseudo-time PBE to overcome the nonlinear difficulty [47]. However, discretizing the current QPBE model yield in the form of

$$
A(\mathbf{U}) \mathbf{U}+N(\mathbf{U})=\mathbf{f},
$$

where the vector $\mathbf{U}$ is the solution of the QPBE. When the Newton's method is applied, it is usually very complicated to have the Jacobian matrix of $A(\mathbf{U}) \mathbf{U}+N(\mathbf{U})$ because of the dependence of $A$ on $\mathbf{U}$. To address this issue, a systematic formulation of the Jacobian matrix for the direct Newton's method will be derived. Additionally, a mixed fixed-point-Newton's method, which requires simpler Jacobian matrix is also proposed as an alternative. Further, this method is more general to solve the QPBE with interface conditions. Efficiency and accuracy of the numerical methods are tested with a set of parameters through two-dimensional and three-dimensional examples.

At last, applications of the proposed QPBE model and computational methods are investigated for biological macromolecules in a complicated solvent. Three examples are provided, including calculation of electrostatic solvation free energy for proteins, description of pore properties of ion channels, and surface electrostatic analysis of a DNA strand. Simulations of the QPBE are performed with a wide set of physiological parameters. Comparing to the classical PBE, the QPBE can provide more physical profiles of electrostatics, ionic concentrations, and a detailed description of dielectric property of solvent near molecular surfaces.

The remaining part of the paper is organized as follows. The mathematical model and the derived governing equation are presented in Section 2. Numerical algorithms of the nonlinear equation are gathered in Section 3. Section 4 is devoted to the numerical analysis of the proposed scheme, and three example applications of the model about solute-solvent system are provide in Section 5. Concluding remarks are given in Section 6.

\section{Mathematical model}

\subsection{Governing equations}

Assuming in the solvent there are totally $N_{c}$ ionic species, whose ionic concentration, reference concentration, and valence are $n_{j}, n_{j}^{0}$ and $q_{j}$, respectively. We consider the free energy functional in terms of $n_{j}$ and the electrostatics $\phi$ in the domain $\Omega$ :

$$
G\left[\phi, n_{1}, \ldots, n_{N_{c}}\right]=\int_{\Omega}\left[k_{B} T \sum_{j=1}^{N_{c}} n_{j} \ln \frac{n_{j}}{n_{j}^{0}}-\frac{\epsilon\left(n_{1}, \ldots, n_{N_{c}}\right)}{8 \pi}|\nabla \phi|^{2}+\phi \rho\right] d \mathbf{r}+\int_{\Omega} k_{B} T \sum_{j=1}^{N_{c}}\left(n_{j}^{0}-n_{j}\right) d \mathbf{r} .
$$

Equation (1) is still in the classical formulation[48, 49]: its first line gives the entropy of mobile ions and electrostatic free energy of the system, with $k_{B}$ and $T$ being the Boltzmann constant and the absolute temperature, respectively. The overall charge density $\rho$ includes fixed charge in the system and the mobile ionic species, i.e.,

$$
\rho=\rho_{f} e+\sum_{j=1}^{N_{c}} n_{j} q_{j}
$$




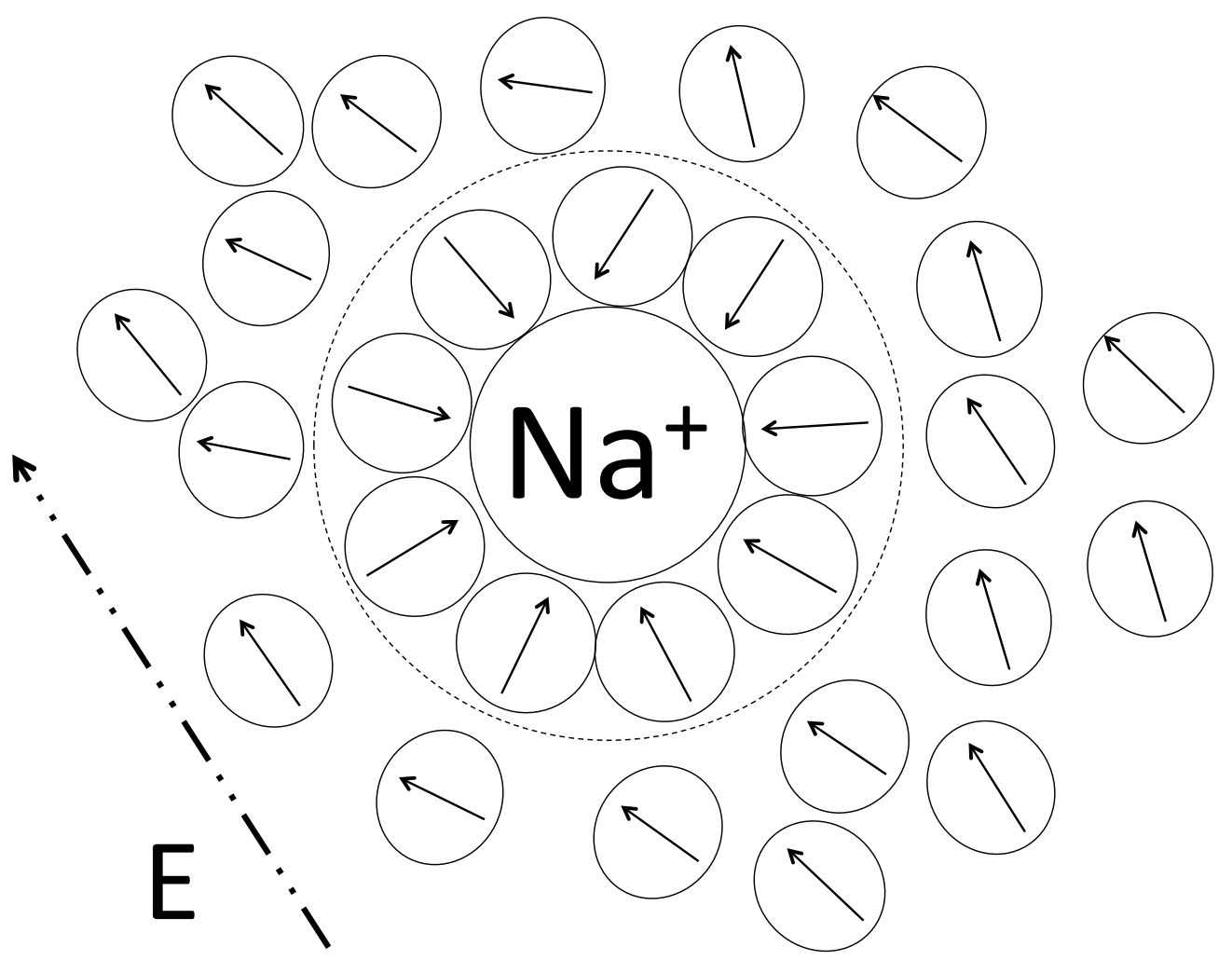

Figure 1: A schematic drawing of ionic concentration dependent dielectric response of water molecules. In vicinity of an ion (e.g. $\mathrm{Na}^{+}$, treated as a point charge but marked as a hard ball in this drawing), water molecules form a hydration shell(dashed circle), and their orientations follow the field lines generated by the cation itself but rather than the local external electrostatic electrostatic filed $E$ (dashed dot arrow line). This effect leads to a heterogeneous dielectric decrease. Solid arrows represent the dipoles of water molecules.

Different from the conventional energy formulation, the dielectric function $\epsilon\left(n_{1}, \ldots, n_{N_{c}}\right)$ in Eq. (1) is taken as a function depending on ionic concentrations instead of a constant. Figure 1 gives the physical explanation: water molecules form a hydration shell around a solvated ion. When away from the ion-water cluster (dashed circle), the orientation of water molecules generally follow the external electrostatic field. In contrast, rotation of those dipoles in the hydration shell are greatly restricted in the vicinity of an ion. They are oriented immediately along the field line generated by the cation or anion, leading to an overall decrease in the dielectric response to the electrostatic field. This decrement depends on ionic concentrations and specific ionic species.

Taking variation of Eq. (1) with respect to $\phi$, we obtain the Poisson equation

$$
\frac{\delta G_{\text {Total }}\left[\phi, n_{1}, \ldots, n_{N_{c}}\right]}{\delta \phi}=0 \Rightarrow-\nabla \cdot\left(\epsilon\left(n_{1}, \ldots, n_{N_{c}}\right) \nabla \phi\right)=4 \pi \rho_{f} e+4 \pi \sum_{j=1}^{N_{c}} n_{j} q_{j},
$$

Equation (2) is not closed and the governing equation for ionic concentration $n_{j}, j=1,2, \ldots, N_{c}$ can be derived by

$$
\frac{\delta G_{\text {Total }}\left[\phi, n_{1}, \ldots, n_{N_{c}}\right]}{\delta n_{j}}=\mu_{j} \Rightarrow \mu_{j}=k_{B} T \ln \frac{n_{j}}{n_{j}^{0}}+q_{j} \phi-\frac{\delta \epsilon}{\delta n_{j}} \frac{|\nabla \phi|^{2}}{8 \pi},
$$

where $\mu_{j}$ is the chemical-electrostatic energy of the $j$-th ionic species. 
Based on the results from experiments and molecular dynamics computation in[42-45], we take

$$
\epsilon\left(n_{1}, n_{2}, \ldots n_{N_{c}}\right)=\epsilon-\sum_{j=1}^{N_{c}} \beta_{j} n_{j},
$$

where $\epsilon$ is the dielectric constant for water and it usually takes value $\epsilon=80$. The parameter $\beta_{j}$ is called the dielectric decrement and its value depends on ionic species and experimental observations. Further, assume the system is in equilibrium $\left(\nabla \mu_{j}=0\right)$, we obtain the modified Boltzmann distribution for the $j$-th ionic species:

$$
n_{j}=n_{j}^{0} \lambda_{j} e^{-q_{j} \phi / k_{B} T}, \quad \text { where } \lambda_{j}=e^{-\beta_{j}|\nabla \phi|^{2} / 8 \pi k_{B} T} .
$$

In the current work, we consider a 1:1 electrolyte, i.e., $N_{c}=2, \beta_{1,2}=\beta / 2$, and $q_{1}=-q_{2}=e$, where $e$ is the elementary charge, then we have

$$
\lambda_{1,2}=\lambda=e^{-\beta|\nabla \phi|^{2} / 16 \pi k_{B} T},
$$

Combining Eqs. (2), (4)-(6), we arrive at a quasi-linear Poisson-Boltzmann equation (QPBE):

$$
-\nabla \cdot(\epsilon(\mathbf{r}) \nabla \phi)+8 \pi n_{0} e \lambda \sinh \left(\frac{\phi e}{k_{B} T}\right)=4 \pi \rho_{f} e,
$$

where

$$
\epsilon(\mathbf{r})=\epsilon-\lambda \beta n^{0} \cosh \left(\frac{\phi e}{k_{B} T}\right)
$$

\subsection{Equation nondimensionalization and parameter estimate}

It is convenient to write the system in a dimensionless form. The scale $(L)$ of the system is several hundreds of angstroms (A) and the unit of ionic concentration is molar (M). So let $\hat{L}=\frac{L}{\AA}, \hat{n}_{0}=\frac{n_{0}}{M}, \hat{\beta}=\beta M, M=$ $\frac{m o l}{\text { liter }}=\frac{N_{A}}{10^{3} \times 10^{24} \AA^{3}}=\frac{N_{A}}{10^{27} \AA^{3}}\left(N_{A}\right.$ is the Avogadro constant $)$ and $u=\frac{\phi e}{k_{B} T}$, Eqs. (7) and (8) are scaled to a nondimensionalized form,

$$
-\nabla \cdot\left[\left(\epsilon-I_{s} \beta e^{-c_{1} \beta|\nabla u|^{2}} \cosh u\right) \nabla u\right]+c_{2} I_{s} e^{-c_{1} \beta|\nabla u|^{2}} \sinh u=c_{3} \rho_{f},
$$

where the constants $c_{1}=\frac{10^{27} k_{B} T \AA}{16 \pi e^{2} N_{A}} \approx 0.06, c_{2}=\frac{8 \pi e^{2} N_{A}}{10^{27} k_{B} T \AA} \approx 8.44$, and $c_{3}=\frac{4 \pi e^{2}}{k_{B} T \AA} \approx 7046$. Notice that the hat is dropped for convenience. Also, only the decrement parameter $\beta$ and the ionic strength $I_{s}=n_{0}$ are kept for different physiological conditions. The range for ionic strength is usually taken as $I_{s}=0.1 \sim 2.0$ and the decrement parameter is $\beta=10 \sim 20$.

\section{Numerical algorithm}

In this Section, we discuss discretization and iteration schemes for the proposed QPBE. For demonstration simplicity, the parameters $c_{1}, c_{2}$ and $c_{3}$ in Eq. (9) are absorbed and the equation

$$
-\nabla \cdot\left[\left(\epsilon-I_{s} \beta e^{-\beta|\nabla u|^{2}} \cosh u\right) \nabla u\right]+I_{s} e^{-\beta|\nabla u|^{2}} \sinh u=f
$$

is considered, while the original equation is used in all numerical implementations. 


\subsection{Discretization}

For simplicity, we assume the computational domain is a rectangular box $\Omega=\left[0, L_{x}\right] \times\left[0, L_{y}\right] \times\left[0, L_{z}\right]$ and a uniform mesh size $h=\frac{L_{x}}{N_{x}}=\frac{L_{y}}{N_{y}}=\frac{L_{z}}{N_{z}}$ is assumed, where $N_{x}, N_{y}$, and $N_{z}$ are total discretization numbers along each direction. Let $\mathcal{T}_{h}=\left\{x_{i}, y_{j}, z_{k}\right\}_{i, j, k=1}^{N_{x}, N_{y}, N_{z}}$ be a mesh of $\bar{\Omega}$ and $U=\left\{u_{i, j, k}\right\}$ be the discretization of the function $u(\mathbf{r})$ on the mesh $\mathcal{T}_{h}$. Denote the standard (forward and backward) first-order difference operators along the $x$-direction by

$$
\delta_{i}^{+} U=\frac{u_{i+1, j, k}-u_{i, j, k}}{h}, \quad \delta_{i}^{-} U=\frac{u_{i, j, k}-u_{i-1, j, k}}{h}
$$

and hence the second-oder scheme

$$
\delta_{i}^{2} U=\frac{\epsilon_{i+\frac{1}{2}, j, k} \delta_{i}^{+}-\epsilon_{i-\frac{1}{2}, j, k} \delta_{i}^{-}}{h} U
$$

along the $x$-direction. The operators $\delta_{j}^{ \pm}, \delta_{k}^{ \pm}, \delta_{j}^{2}$ and $\delta_{k}^{2}$ along the $y$ - and $z$-directions can be denoted similarly. In Eq. (12), we have

$$
\epsilon_{i+\frac{1}{2}, j, k}=\epsilon-I_{s} \beta \exp \left[-\beta \sum_{m=i, j, k}\left(\delta_{m}^{\star,+} U\right)^{2}\right] \cosh \left(\delta_{i}^{0,+} U\right)
$$

and

$$
\epsilon_{i-\frac{1}{2}, j, k}=\epsilon-I_{s} \beta \exp \left[-\beta \sum_{m=i, j, k}\left(\delta_{m}^{\star,-} U\right)^{2}\right] \cosh \left(\delta_{i}^{0,-} U\right) .
$$

Since the discretization of $\epsilon_{i+\frac{1}{2}, j, k}$ at the point $\left(i+\frac{1}{2}, j, k\right)$ is off the mesh $\mathcal{T}_{h}$, we introduce $\delta_{m}^{\star,+}, m=i, j, k$ as the difference operators to approximate $u_{x}, u_{y}$ and $u_{z}$ at the point, while $\delta_{i}^{0,+}$ represents the interpolation of $u$ there (along the $x$-directoin). All these coefficients can be easily obtained and are subject to second-order local truncation error. Similar notations $\delta_{m}^{\star,-}$ and $\delta_{m}^{0,-}$ are adopted for $\epsilon(\mathbf{r})$ at the point $\left(i-\frac{1}{2}, j, k\right)$. The finite difference operator along $y$ - and $z$ - directions are defined analogically and thus Eq. (10) is discretized at the grid point $(i, j, k)$ as

$$
-\sum_{m=i, j, k} \delta_{m}^{2} U+I_{s} \exp \left[-\beta \sum_{m=i, j, k}\left(\frac{\delta_{m}^{+}+\delta_{m}^{-}}{2} U\right)^{2}\right] \sinh \left(\delta_{i j k} U\right)=f_{i j k},
$$

where the notation $u_{i, j, k}=\delta_{i j k} U$ is adopted for convenience in later description.

\subsection{Iteration methods}

Equation. (15) can be written in a matrix form

$$
A(U) U+N(U)=\mathbf{f},
$$

where

$$
A(U)=-\sum_{m=i, j, k} \delta_{m}^{2},
$$

and it depends on $U$ and $\delta U$ according to Eqs. (12)-(14). Meanwhile,

$$
N(U)=I_{S} \exp \left[-\beta \sum_{m=i, j, k}\left(\frac{\delta_{m}^{+}+\delta_{m}^{-}}{2} U\right)^{2}\right] \sinh \left(\delta_{i j k} U\right) .
$$

The major difficulty of solving Eq. (16) is the nonlinearity of the matrix $A$ on $U$, so we need to look for efficient method to solve the nonlinear algebra system (16) iteratively. 


\subsubsection{Jacobian matrix for the Newton's method}

Equation (16) can be solved by the classical Newton's method. In the traditional PBE, the differential operator $A$ has no dependence on the solution $U$ and the nonlinear part $N(U)$ is diagonal, so the Jacobian matrix is very easy to compute. But for the QPBE, calculation of $J(U)$ is not trivial. Here we present a systematic way to calculate the Jacobian matrix based on the notation introduce above.

First of all, the Jacobian matrix can be formed as

$$
J(U)=\frac{\partial}{\partial U}[A(U) U+N(U)]=A(U)+\frac{\partial}{\partial U} A(U) U+\frac{\partial}{\partial U} N(U),
$$

Then the second term of the right hand side of Eq. (17), according to Eq.(16), is

$$
\frac{\partial}{\partial U} A(U)=-\sum_{m=i, j, k} \frac{\partial}{\partial U} \delta_{m}^{2} U
$$

For simplicity, we only carry out $\frac{\partial}{\partial U} \delta_{i}^{2}$ in Eq. (18). Recalling Eqs. (12)-(14), we have

$$
\frac{\partial}{\partial U} \delta_{i}^{2}=\frac{\partial}{\partial U} \frac{\epsilon_{i+\frac{1}{2}, j, k} \delta_{i}^{+}-\epsilon_{i-\frac{1}{2}, j, k} \delta_{i}^{-}}{h}=\frac{\partial \epsilon_{i+\frac{1}{2}, j, k}}{\partial U} \frac{\delta_{i}^{+}}{h}-\frac{\partial \epsilon_{i-\frac{1}{2}, j, k}}{\partial U} \frac{\delta_{i}^{-}}{h} .
$$

Further,

$$
\begin{aligned}
\frac{\partial \epsilon_{i+\frac{1}{2}, j, k}}{\partial U} & =-I_{S} \exp \left[-\beta \sum_{m=i, j, k}\left(\delta_{m}^{\star,+} U\right)^{2}\right] \sinh \left(\delta_{i}^{0,+} U\right) \delta_{i}^{0,+} \\
& -I_{S} \exp \left[-\beta \sum_{m=i, j, k}\left(\delta_{m}^{\star,+} U\right)^{2}\right] \cosh \left(\delta_{i}^{0,+} U\right) \times\left(-2 \beta \sum_{m=i, j, k} \delta_{m}^{\star,+} U \cdot \delta_{m}^{\star,+}\right) \\
& =2 I_{S} \beta\left(\sum_{m=i, j, k} \delta_{m}^{\star,+} U\right) \exp \left[-\beta \sum_{m=i, j, k}\left(\delta_{m}^{\star,+} U\right)^{2}\right] \cosh \left(\delta_{i}^{0,+} U\right) \delta_{m}^{\star,+} \\
& -I_{S} \exp \left[-\beta \sum_{m=i, j, k}\left(\delta_{m}^{\star,+} U\right)^{2}\right] \sinh \left(\delta_{i}^{0,+} U\right) \delta_{i}^{0,+},
\end{aligned}
$$

and

$$
\begin{aligned}
\frac{\partial \epsilon_{i-\frac{1}{2}, j, k}}{\partial U} & =2 I_{s} \beta\left(\sum_{m=i, j, k} \delta_{m}^{\star,-} U\right) \exp \left[-\beta \sum_{m=i, j, k}\left(\delta_{m}^{\star,-} U\right)^{2}\right] \cosh \left(\delta_{i}^{0,-} U\right) \delta_{m}^{*,-} \\
& -I_{s} \exp \left[-\beta \sum_{m=i, j, k}\left(\delta_{m}^{\star,-} U\right)^{2}\right] \sinh \left(\delta_{i}^{0,-} U\right) \delta_{i}^{0,-} .
\end{aligned}
$$

Note that the operators $\delta_{i}^{ \pm}$in Eq. (19) are acting on $U$, while $\delta_{m}^{\star, \pm}$ and $\delta_{i}^{0, \pm}$ in Eqs. (20)-(21) are acting on the error vector in the Newton's method.

In a similar fashion, we can derive

$$
\begin{aligned}
\frac{\partial}{\partial U} N(U) & =-I_{s} \beta\left[\sum_{m=i, j, k}\left(\delta_{m}^{+}+\delta_{m}^{-}\right) U\right] \exp \left[-\beta \sum_{m=i, j, k}\left(\frac{\delta_{m}^{+}+\delta_{m}^{-}}{2} U\right)^{2}\right] \sinh \left(\delta_{i j k} U\right) \times \frac{1}{2} \sum_{m=i, j, k}\left(\delta_{m}^{+}+\delta_{m}^{-}\right) \\
& +I_{s} \exp \left[-\beta \sum_{m=i, j, k}\left(\frac{\delta_{m}^{+}+\delta_{m}^{-}}{2} U\right)^{2}\right] \cosh \left(\delta_{i j k} U\right) \delta_{i j k}
\end{aligned}
$$

Combining Eqs. (17)-(22), the Newton's method for solving the QPBE can be outlined as: 
- Compute the residue vector $\mathbf{r}^{p}: \mathbf{r}^{p}=\mathbf{f}-A\left(U^{p}\right) U^{p}-N\left(U^{p}\right)$, assuming the solution $U^{p}$ at the $p$-th step is available;

- Compute the error vector $\mathbf{e}^{p}$ by solving the linear system $J\left(U^{p}\right) \mathbf{e}^{p}=\mathbf{r}^{p}$, where $J\left(U^{p}\right)$ is the Jacobian matrix of $A\left(U^{p}\right) U^{p}+N\left(U^{p}\right)$

- Update $U^{p}$ to $U^{p+1}$ by $U^{p+1}=U^{p}+\mathbf{e}^{p}$ and check the convergence.

\subsubsection{The fixed-point-Newton's method}

As stated above, calculating the Jacobian matrix in the standard Newton's method is complicated. In some cases, as we can see in Section 5, where the $\epsilon(\mathbf{r})$ is not continuous in $u$, direct computing Eqs. (20) and (21) could be problematic. Hence we propose an alternative way, the fixed-point-Newton's method, to solve the QPBE in a simpler form and for more general cases.

Assume $K_{s}$ to be the solution space of the QPBE and consider the fixed point problem $u^{\star}=\mathcal{F}(u)$, where the map $\mathcal{F}: K_{s} \rightarrow K_{s}$ is defined implicitly by

$$
-\nabla \cdot\left[\left(\epsilon-I_{s} \beta e^{-\beta|\nabla u|^{2}} \cosh u\right) \nabla u^{\star}\right]+I_{s} e^{-\beta|\nabla u|^{2}} \sinh u^{\star}=f .
$$

In practice, we seek the fixed point by the steady-state problem of the following ordinary differential equation (ODE)

$$
\frac{\partial u}{\partial t}=u^{\star}-u=\mathcal{F}(u)-u,
$$

thus various ODE-related techniques such as Runge-Kutta method can be used. One simple implementation is

$$
\frac{u^{n+1}-u^{n}}{\alpha}=\mathcal{F}\left(u^{n}\right)-u^{n}=u^{\star}, n-u^{n}, \quad n=1,2, \ldots
$$

which leads to a self-consistent iteration with a relaxation factor $\alpha$

$$
u^{n+1}=\alpha u^{\star}, n+(1-\alpha) u^{n} .
$$

With given $u^{n}$, the unknown $u^{\star, n}$ is solved through Eq. (23) by the standard Newton's method, then we can proceed to $u^{n+1}$ by Eq. (25) and check the convergence. Note that in this case the Jacobian matrix of Eq. (23) reads:

$$
J\left(U, U^{\star}\right)=\frac{\partial}{\partial U^{\star}}\left[A(U) U^{\star}\right]+\frac{\partial}{\partial U^{\star}}\left[N\left(U^{\star}\right)\right] .
$$

The first term of the right hand side of Eq. (26) is $A(U)$, which only depends on the solution in the previous step, so differentiating the matrix with respect to the solution in the current step is avoid. This treatment simplifies the Jacobian matrix calculation and is more suitable for the case when $A(U)$ is not continuos with respect to $U$. The tradeoff is that this method is a multi-iterative scheme: the iteration (25) can be viewed as the outer loop, while solving the nonlinear equation (23) is the inner loop.

\section{Numerical results}

In this section, Eq. (9) is solved to test the computational efficiency and accuracy of the proposed numerical algorithms. The comparisons of solutions of the QPBE and the traditional PBE are also investigated.

\subsection{Computational efficiency and accuracy}

We consider the equation in 2D on the domain $\Omega=[0,1] \times[0,1]$ and take the Dirichelet boundary condition $u=g$ on $\partial \Omega$. To test the iterative efficiency of the proposed algorithms, we fix $N_{x}=N_{y}=101$ and assume $f=0$ for simplicity, while the parameters $I_{s}, \beta$, and $g$ are varied. 


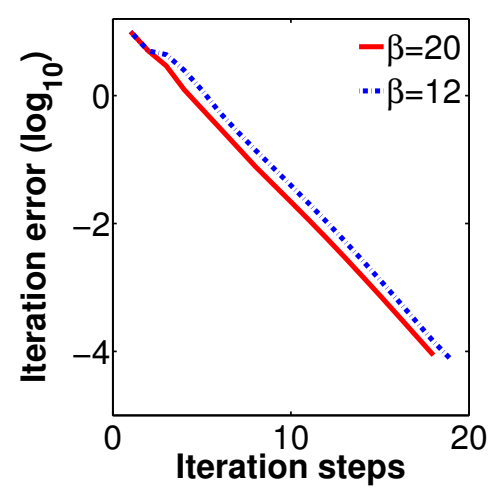

(a)

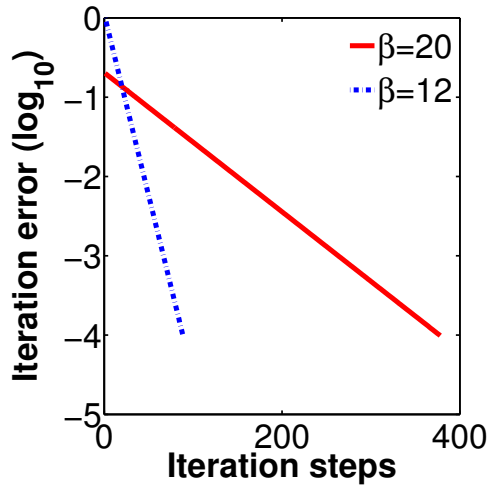

(b)

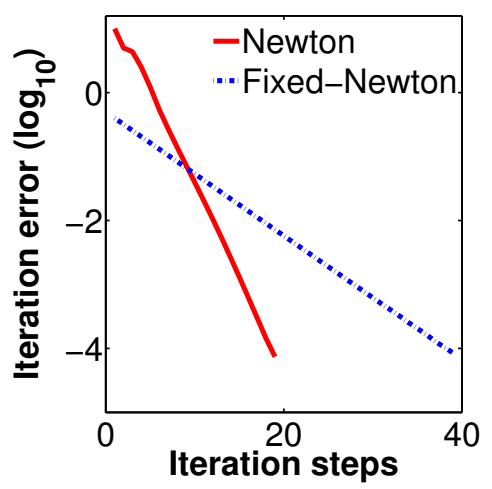

(c)

Figure 2: Computational efficiency of the Newton's method and the fixed-point-Newton's method. (a): Newton's method; (b) Fixed-point-Newton's method. For (a) and (b), $I_{S}=0.2, g=-10$. (c): comparison of the two method with $I_{s}=0.2, \beta=12$ but $g=-2$.

Figure 2 shows iterative efficiency of the Newton's method and the fixed-point-Newton's method. The horizontal axis shows the iteration step $n$ and the vertical axis marks the $\log _{10}$ of the iteration error $\| u^{n+1}$ $u^{n} \|_{L^{\infty}}$.

First we set $g=-10, I_{s}=0.2$ and choose different values of $\beta$. For the full Newton's method, the iteration efficiency is not affected too much by the choice of $\beta$ : as shown in Fig. 2 (a), when $\beta=12$ and 20, the iterative errors reduce to the designated criteria $\left(1.0 \times 10^{-4}\right)$ within 20 steps. In contrast, the fixed-pointNewton's method needs much more iterative steps $(\sim 100)$ to achieve the same iterative error, as shown in Fig. 2(b). Further, when the parameter $\beta$ increases from 12 to 20, the QPBE appears a "stronger" nonlinearity and the iterative step increases from 100 to 400 . We conclude that the fixed-point-Newton's method promises a much simpler discretization scheme but yields less computational efficiency. But this scheme is still useful in complicated applications.

With the same ionic strength and $\beta=12$, Fig. 2(c) shows the comparison of the two iterative methods when $|g|$ is relatively small. It can be concluded that there is no serious difference between the efficiencies of the two methods when the boundary condition is taken as $g=-2$. Iteration step of the Newton's method does not heavily depends on these parameters; it is still around 20 as in Fig. 2 (a). But the fixed-Point-Newton's method requires significantly less iterative steps in this case.

From another point of view, CPU time of the two methods are listed in Table 1. The code was run on an iMac desktop with $3.4 \mathrm{GHz}$ Intel Core i7 processor and 32GB memory. The unit of CPU time in the table is second. The conclusion in terms of time is similar to that from Fig. 2: for all kinds of parameter settings, the CPU time of the Newton's method is around 0.03 second, while the time used by the fixed-point-Newton's method depends on the specific parameters and is longer than those from Newton's method.

Table 1: CPU time (unit: second) of the Newton's method and the fixed-point-Newton's (F-N)method under different parameter settings.

\begin{tabular}{l|l|l}
\hline & Newton's method & F-N Method \\
\hline$g=-2, \beta=12$ & $3.0 \mathrm{e}-2$ & $2.4 \mathrm{e}-1$ \\
$g=-10, \beta=12$ & $3.7 \mathrm{e}-2$ & $6.6 \mathrm{e}-1$ \\
$g=-10, \beta=20$ & $3.7 \mathrm{e}-2$ & 1.28 \\
\hline
\end{tabular}


Although the analysis of the two iterative methods remains open, numerically we are interested in whether the two iterations converge to the same solution. The numerical solution of Eq. (9) at a very fine grid $N_{x}=N_{y}=161$ is considered as the reference solution $u^{0}$, and the error $\left\|u-u^{0}\right\|_{L_{\infty}}$ is calculated at the grid $N_{x}=N_{y}=11,21,41$ and 81. Other parameters are taken as $g=-10, I_{s}=0.2$, and $\beta=20$.

Table 2: Convergence rates of the Newton's method (first two columns) and the fixed-point-Newton's (F-N)method (the third and fourth columns). The relative differences of the solutions from the two methods are in the last column.

\begin{tabular}{ll|ll|l}
\hline $\begin{array}{l}\text { Newton's method } \\
\text { Error }\end{array}$ & Order & F-N Method & Relative difference \\
\hline $5.25 \mathrm{e}-5$ & & $5.26 \mathrm{e}-5$ & Order & \\
$1.29 \mathrm{e}-5$ & 2.0 & $1.299 \mathrm{e}-5$ & 2.0 & $0.07 \%$ \\
$3.09 \mathrm{e}-6$ & 2.0 & $3.09 \mathrm{e}-6$ & 2.0 & $0.07 \%$ \\
$6.18 \mathrm{e}-7$ & 2.3 & $6.19 \mathrm{e}-7$ & 2.3 & $0.07 \%$ \\
\hline
\end{tabular}

Table 2 lists the numerical errors and convergence orders for the two algorithms. The convergence order is calculated as $\log _{2} \frac{\left\|u^{2 h}-u^{0}\right\|_{L_{\infty}}}{\left\|u^{h}-u^{0}\right\|_{L_{\infty}}}$, where $u^{h}$ is the numeral solution of Eq. (9) at the grid space $h=\frac{1}{N_{x}-1}$. Both of the two iteration methods can achieve second-order convergency and the last column of Table 2 shows that the relative differences of solutions from the two iterative methods are within $0.1 \%$.

\subsection{Comparison with Poisson-Boltzmann equation}

In this section, Eq. (9) is solved in 1D by the Newton's method with a set of parameter combinations and the physical profiles are presented to illustrate the differences from the PBE model. The domain is set as [0,1] and discretized with $N_{x}=201$. Additionally, different Dirichlet boundary conditions are applied for $u(0)$, while the Neumann boundary condition $u_{x}(1)=0$ is taken for all the cases.

Figure 3 displays the electrostatic potential (red) and ionic concentrations (blue) calculated from the PBE and QPBE with $I_{s}=0.2$ and $\beta=12$. Two cases (left and right column), a lightly charged boundary $u(0)=-2$ and a boundary with relatively heavy charges $u(0)=-10$ are compared. The horizontal axis shows the distance from the left charged boundary $x=0$, in the unit of Angstrom and the vertical axis displays the electrostatics in the unit of $k_{B} T / e$ or ionic concentration in the unit of molar. Figs 3(a) and 3(c) suggest that when the boundary is lightly charged, the differences of solutions between the PBE and QPBE models are not significant for both electrostatics and ionic concentrations. However, when the charge on the boundary is increased to $u(0)=-10$, the results are remarkably different. In Figs. 3(b), the electrostatics calculated from the PBE decays rapidly because of the screening effects, while the decay is obviously alleviated in the QPBE model. In the PBE model, the calculated ionic concentration near the boundary yields un-physically large quantity, as seen in the inset of Fig. 3(d), where the concentration goes to $2 \times 10^{4} \mathrm{M}$; on the other hand, the concentration calculated from the QPBE is well-controlled near the boundary.

In many applications, the boundary is strongly charged, so we are specifically interested in the case of $u(0)=-10$. Fig. 4 shows the simulations of QPBE with various combinations of parameters $I_{s}$ and $\beta$. The left column display the results for a weak ionic strength $\left(I_{s}=0.2\right)$, while the right column are for a relatively stronger one $\left(I_{s}=2.0\right)$. In each category the simulations are performed with $\beta=10$ and $\beta=20$. We conclude that when the value of $\beta$ is doubled, there is a minor change in electrostatics but the parameter has a more significant effect on ionic concentration. More interestingly, the green dashed curve in Fig. 4(d) suggests that when the surface charge density and reference ionic strength are relatively large $\left(u(0)=-10\right.$ and $\left.I_{S}=2.0\right)$, an increased value of $\beta$ may introduce a non-monotone ionic concentration profile near the boundary. This is an essentially different phenomenon from the standard PB model. It has potential applications in biomolecules in salt-solvent and is worth verification by biological experiments. 


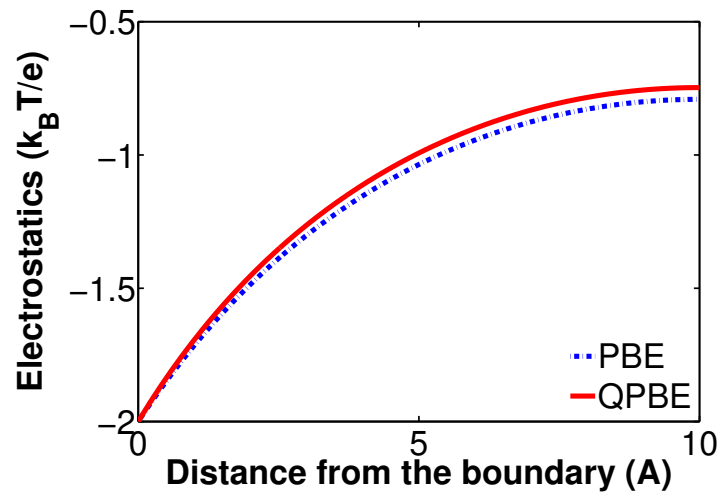

(a)

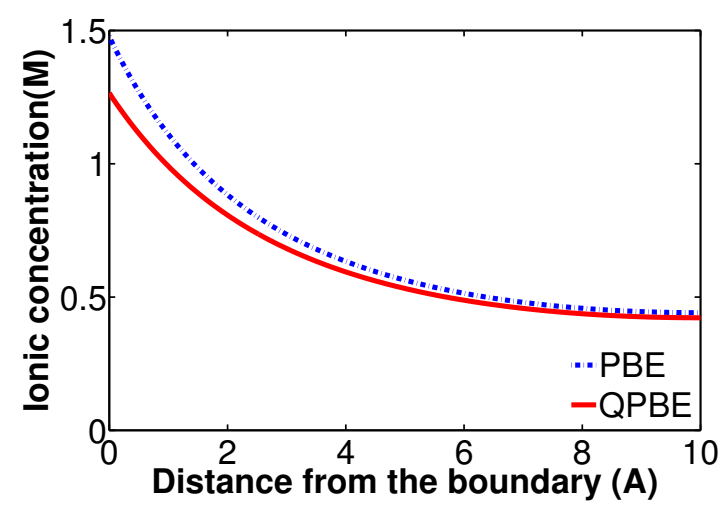

(c)

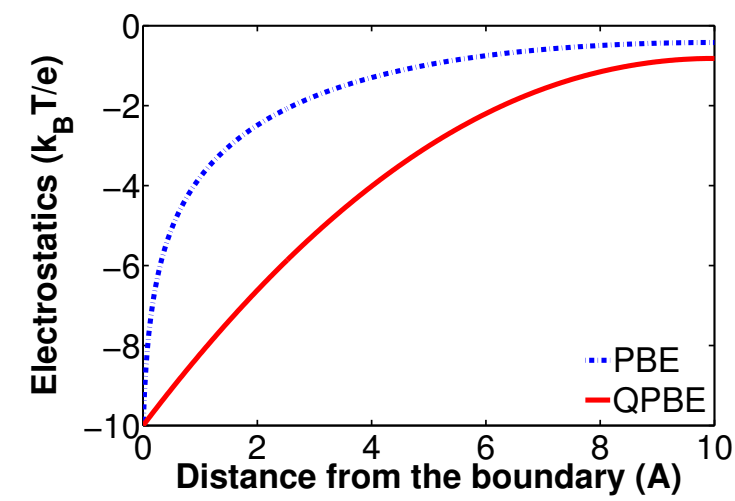

(b)

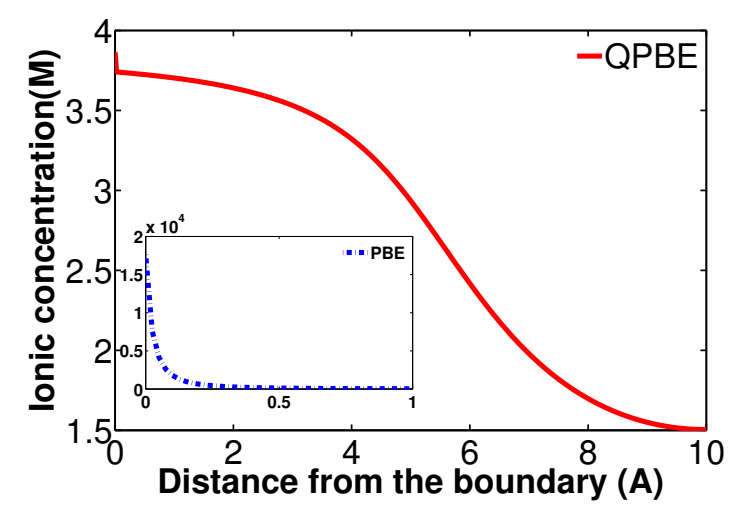

(d)

Figure 3: Comparison of physical profiles computed by the PBE and the QPBE. First column: boundary condition $u(0)=-2$; second column: boundary condition $u(0)=-10$. First row: electrostatics; second row: ionic concentration. In these simulations, $I_{S}=0.2$ and $\beta=12$.

Figure 5 displays the simulated heterogeneous dielectric function near the charged boundary. It can be concluded that the dielectric permittivity depends on the boundary condition, $I_{s}$ and $\beta$. Combination of larger values from $I_{s}$ and $\beta$ gives more significant dielectric decrement from the constant 80 . We notice that with boundary condition $u(0)=-10, \beta=20$, and $I_{s}=2.0$, the dielectric of the solvent can be reduce to below $10 \epsilon_{0}$ around $10 A$ (the purple curve). The reason is that, according to the result in Fig. 4(d), ions concentrate in $10 \mathrm{~A}$ from the charged boundary. Whether this is the correct physical picture is still not clear. It is very meaningful to discuss in the future work about the physical justification of such computational results and thus for the proper parameter ranges of the current QPBE model.

\section{Applications in molecular biosciences}

In this section we present several applications of the QPBE model in molecular biosciences. Figure 6 shows the schematic diagram of the heterogeneous dielectric implicit solvent model for the biomacromolecules in an ionic solvent. Biomolecules (proteins, DNAs or RNAs) are described in the domain $\Omega^{+}$at the atomic scale with their three dimensional structures: the positions, charges, and radii of atoms are obtained from the Protein Data Bank(PDB) . On the contrast, water molecules are modeled as dielectric continuum and mobile ions in 


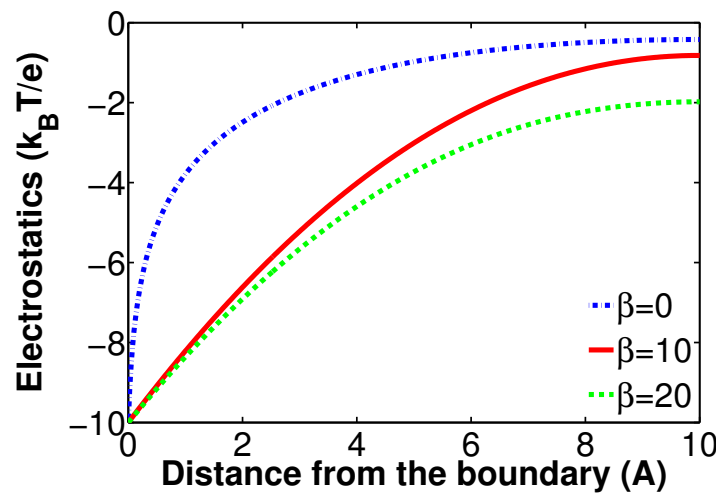

(a)

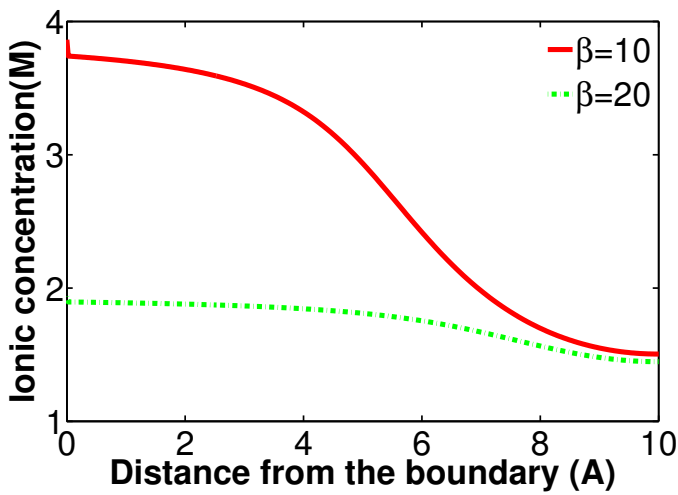

(c)

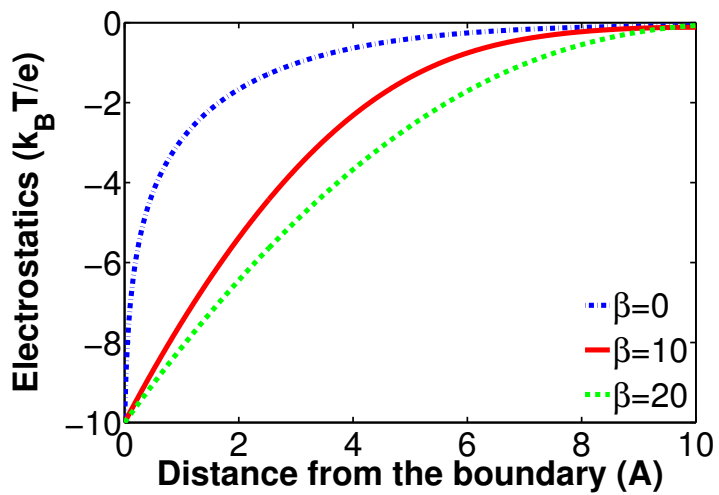

(b)

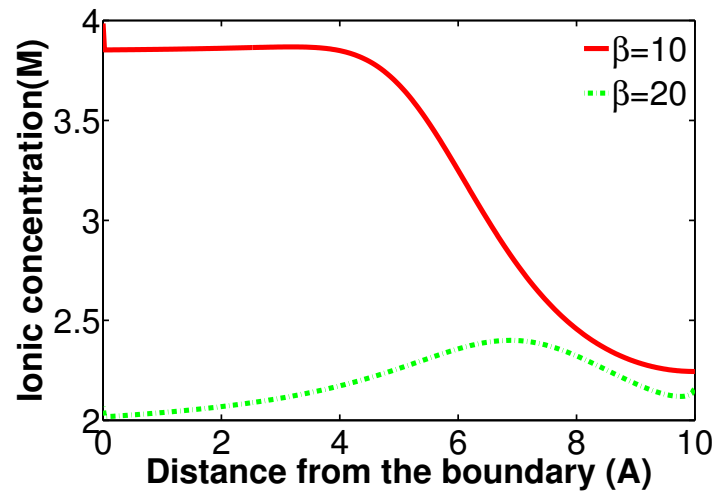

(d)

Figure 4: Comparison of physical profiles computed by the QPBE with different sets of parameters $I_{S}$ and $\beta$. First column: $I_{S}=$ 0.2 ; second column: $I_{S}=1.5$. First row: electrostatics; second row: ionic concentration. In these simulations, the boundary condition is taken as $u(0)=-10$.

the solvent domain $\Omega^{-}$are considered in terms of concentrations. The solute domain $\Omega^{+}$and solvent domain $\Omega^{-}$are separated by the molecular surface $\Gamma$.

The proposed QPBE is used to calculate the electrostatics for the bimolecular system, but two difficulties need to be addressed in computations. One is the piece-wisely defined dielectric function in the domain $\Omega=$ $\Omega^{+} \cup \Omega^{-}$, i.e.

$$
\epsilon(\mathbf{r})= \begin{cases}\epsilon^{+}, & \mathbf{r} \in \Omega^{+}, \\ \epsilon^{-}-I_{S} \beta e^{-c_{1} \beta|\nabla u|^{2}} \cosh u, & \mathbf{r} \in \Omega^{-},\end{cases}
$$

where $\epsilon^{+}$is the dielectric constant for vacuum $\left(\epsilon^{+}=1\right)$ and $\epsilon^{-}=80$ for water. Thus, the function $\epsilon(\mathbf{r})$ is discontinuous across the interface $\Gamma$.

Another issue is the fixed charge in the protein, which gives the singular charge source

$$
\rho_{f}=\sum_{i=1}^{N_{a}} z_{i} \delta\left(\mathbf{r}-\mathbf{r}_{i}\right)
$$

where $z_{i}$ and $\mathbf{r}_{i}$ are the charge fraction and position of the $i$-th atom. Although regular smooth functions such as Gaussion functions can be used to approximate the delta functions, analytic or more accurate treatments of the singularity are desired. 


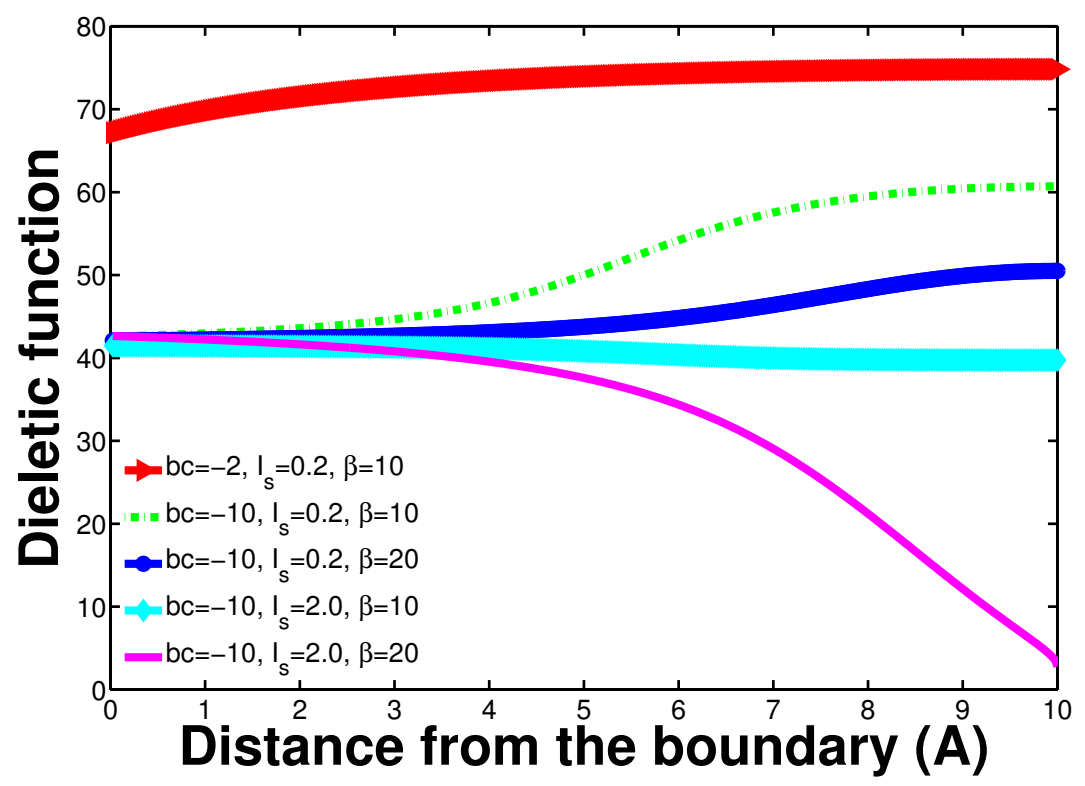

Figure 5: Simulations of the dielectric functions from the QPBE with various parameters: boundary condition (bc), ionic strength $\left(I_{S}\right)$, and decrement parameter $(\beta)$.

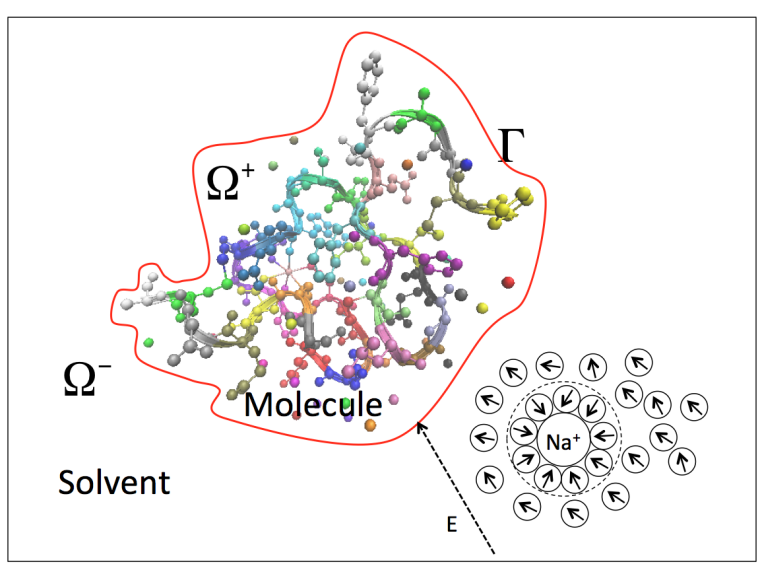

(a)

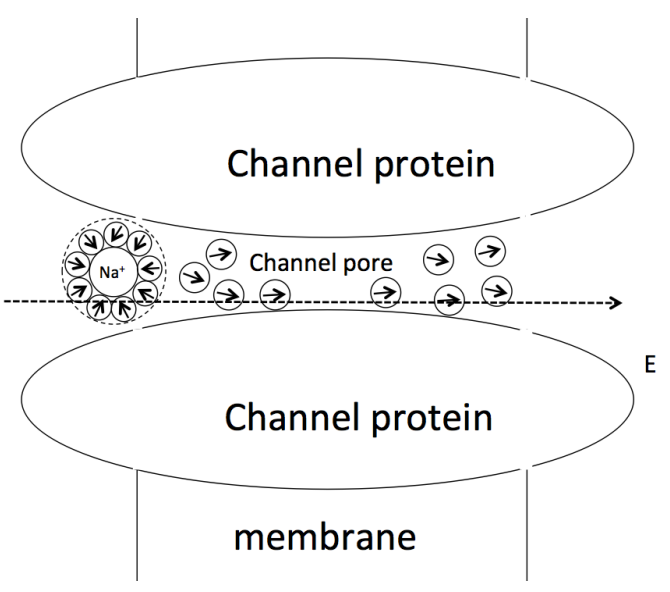

(b)

Figure 6: (a) A schematic drawing of application of the QPBE in the implicit protein-solvent model for biomolecules. Molecular surface $\Gamma$ partitions the whole region into the solute part $\left(\Omega^{+}\right)$and the solvent part $\left(\Omega^{-}\right)$. Atomic description of molecular structure is given for the solute, while the solvent is considered as a continuum with heterogeneous dielectric response. (b) A schematic drawing of the nonhomogeneous dielectric decrement in the pore of an ion channel. The 3D structure of the channel protein is omitted in the graph.

To handle the above difficulties, the matched interface and boundary (MIB) method and related algorithms are used, which include proper definition of the interface $\Gamma$, a highly accurate and efficient interface solver, and the Dirichlet to Neumann (DtN) mapping removing singularities [17, 37, 50, 51]. To summarize, the solution $u$ is decomposed as

$$
u(\mathbf{r})=\tilde{u}(\mathbf{r})+u^{*}(\mathbf{r})+u^{0}(\mathbf{r})
$$


where the definitions of $u^{\star}, u^{0}, \bar{u}$, and construction of the interface $\Gamma$ are described in the Appendix. Then we need to solve a regularized interface problem:

$$
\begin{aligned}
& -\nabla \cdot(\epsilon(\mathbf{r}) \nabla \tilde{u}(\mathbf{r}))+c_{2} I_{S} e^{-c_{1} \beta|\nabla \tilde{u}|^{2}} \sinh \tilde{u}=0 \\
& {[\tilde{u}]_{\Gamma}=0} \\
& {\left.[\epsilon \nabla \tilde{u} \cdot \overrightarrow{\mathbf{n}}]\right|_{\Gamma}=-\left.[\epsilon \bar{u} \cdot \overrightarrow{\mathbf{n}}]\right|_{\Gamma}}
\end{aligned}
$$

with (27), by the proposed fixed-point-Newton's iteration, since the dielectric function is not continuous in $\tilde{u}$.

\subsection{Application 1: Calculation of electrostatic solvation free energy}

One of the most important applications of implicit solvent models is to calculate the electrostatic solvation free energy $\Delta G_{\text {elec }}$, which is obtained by summing up all the fixed charges $z_{i}$ in Eq. (28) of the solute, weighted by the reaction field potential $u_{\mathrm{rf}}(\mathbf{r})$. According to the splitting of the overall potential function Eq. (29), we have $u_{\mathrm{rf}}(\mathbf{r})=u^{0}(\mathbf{r})+\tilde{u}(\mathbf{r})$ and the electrostatic solvation free energy is calculated as[17]:

$$
\Delta G_{\text {elec }}=\frac{1}{2} \sum_{i=1}^{N a} z_{i}\left[u^{0}\left(\mathbf{r}_{i}\right)+\tilde{u}\left(\mathbf{r}_{i}\right)\right] .
$$

Table 3 lists the electrostatic solvation free energies for a set of 17 protein molecules, by solving both the PBE

Table 3: Comparison of electrostatic solvation energy (unit: $\mathrm{kcal} / \mathrm{mol}$ ) calculated by the PBE and the QPBE for a set of proteins. $I_{S}=0.2$ and $\beta=12$.

\begin{tabular}{l|llr}
\hline Protein ID & PBE & QPBE & Difference \\
\hline 1AJJ & -1337.34 & -1334.73 & 2.61 \\
1AJK & -1322.24 & -1319.95 & 2.29 \\
1AL1 & -692.08 & -690.5 & 1.58 \\
1BBL & -1368.0 & -1355.0 & 13.0 \\
1BOR & -1122.83 & -1118.50 & 4.33 \\
1BPI & -1825.73 & -1821.21 & 4.52 \\
1CBN & -494.63 & -493.48 & 1.15 \\
1FCA & -1486.81 & -1475.06 & 11.75 \\
1FXD & -3643.70 & -3640.71 & 2.29 \\
1HPT & -1231.29 & -1225.98 & 5.31 \\
1PTQ & -1237.31 & -1235.02 & 2.29 \\
1R69 & -1553.86 & -1552.00 & 1.86 \\
1UXC & -1600.44 & -1595.01 & 5.43 \\
1VII & -1210.59 & -1204.20 & 6.39 \\
1YSN & -1480.50 & -1477.51 & 2.99 \\
2ERL & -115.06 & -1114.28 & 0.78 \\
2PDE & -1047.46 & -1045.88 & 1.58 \\
\hline
\end{tabular}

and the QPBE. We can conclude that the differences of electrostatic solvation free energies calculated from the PBE and the QPBE are very small, for most of the cases they are less than $1 \%$. This is understandable because the modification of the PBE to the QPBE only takes place in the solvent region $\left(\Omega^{-}\right)$but the calculation of electrostatic solvation free energies is on the site of fixed charges of the protein, or in the domain $\Omega^{+}$. The simulations also show that the electrostatic free energy obtained by the QPBE model is generally higher. 


\subsection{Application 2: Pore properties of ion channels}

Ion channels are a special type of membrane proteins that have holes down in the middle. Conducting ionic flux through cell membranes, they are critical for many physiological processes such as action potential generation, nerve or muscle excitation, and signal transductions. Functions of ion channels are heavily determined by the protein structure, electrostatics, and dynamics of ionic flow in the pore region. The narrow channel pore is wrapped up by the dielectric boundary, see Fig. 6(b), hence the orientation of water molecules in that area are strongly subjected to the local electrostatic field but rather than free rotation. So it is critical to model the dielectric property and the corresponding consequences in the channel pore. Most existing meanfiled models, including the PBE, Poisson-Nernst-Planck (PNP) equation, all treat the solvent in the channel pore in a homogenous way. Rigorous treatment of waters in the channel pore requires $a b$ initio quantum dynamics or molecular dynamics, which are limited by expensive computational costs; on the other hand, all the existing implicit solve models completely neglect these interactions since water molecules are treated as structureless and constant dielectric continuum. Alternatively, the proposed QPBE provides a way to model the anisotropic dielectric properties of water molecules in channel pores in a mean field approach.

As an example, physical properties of the Gramicidin A (gA) channel (PDB: 1MAG) are simulated by the QPBE and several profiles in its channel pore are shown in Fig. 7. Since most the physiological conditions contain $\mathrm{Na}^{+}$or $\mathrm{K}^{+}$, a moderate value $\beta=12$ is taken and two different ionic strength $I_{s}=0.1$ (green dashed line) and $I_{s}=1.0$ (red solid line) are adopted in these calculations. It can be concluded from Fig. 7 (a) that at the positions of $x= \pm 9 \mathrm{~A}$, there are two potential wells, where cations are usually attracted. In sense of geometry, as shown in Fig. 7 (b), the channel pore has smallest areas of the cross-section along the channel direction at the two binding sites. Correspondingly, ionic density of cations peak at this two places, as seen in Fig. 7(c). Dielectric properties in the channel pore are crucial to study the dynamics of ion permeation. In the standard Poisson-Nernst-Planck (PNP) model, the dielectric constant in the Poisson equation is usually phenomenologically reduced to a certain constant lower than 80 based on the argument that freedom of water molecules is greatly limited in the narrow geometry. In contrast, the QPBE is able to offer a systematic way to compute the heterogeneous dielectric function. For the gA protein, two sample dielectric functions are calculated by the QPBE with ionic strength $I_{s}=0.1$ and $I_{s}=1.0$, and the results are shown in Fig. 7 (d). It can be conclude that the reduction of dielectric response of water molecules does not only dependent on channel geometries (otherwise Fig. 7(b) and (d) should be similar). Instead, it is a combinational effects of channel narrowness, local electrostatics, and local ionic concentrations. Although still an approximation, this ionic concentration-dependent dielectric function can be extend to non-equilibrium states beyond the QPBE and simulation will give more insightful physical demonstration than the traditional PNP theory.

\subsection{Application 3: Electrostatics calculation of a DNA segment}

Functions of nucleic acids such as DNA or RNA, including genome packaging, protein binding, RNAzyme and DNAzyme activities, are key to all known living organisms and many viruses. Ionic solvent plays a significant role because the counter ions in the solvent prompt appropriate folding and function of DNA/RNA by partially neutralizing the negatively charged phosphate groups in the nucleic acids. The PB theory is naturally a mean-field theory to study the interactions between the DNA/RNA and its surrounding "ion atmosphere". Since the nucleic acids are strongly charged, it is worthwhile to taking into account the inhomogeneous dielectric response of the solvent near the molecular surface, in order to give a more accurate description of electrostatics and ionic distribution near the DNA/RNA.

As the third application, the QPBE is used to calculate electrostatics for the segment of a DNA strand (PDB code: 1D65) with four sets of parameters. A weak $\left(I_{s}=0.2\right)$ and a strong $\left(I_{s}=1.5\right)$ ionic solvent are considered, and the parameters $\beta=12$ and $\beta=20$ represent different type ionic solvents for each case. For a better comparison, differences between the computational results from the QPBE and the standard PBE, $u^{\mathrm{QPBE}}-u^{\mathrm{PBE}}$, with the parameter combinations are mapped to the molecular surface of the biomolecule and shown in Fig. 8. In this figure, the red color represents negative while blue is for positive electrostatics. We 


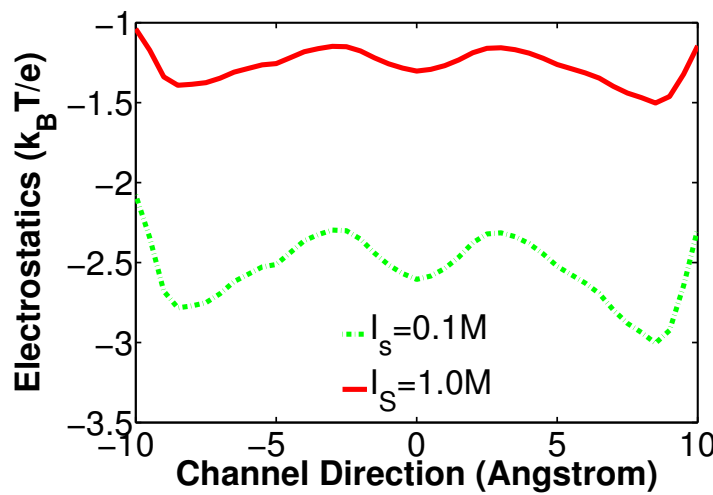

(a)

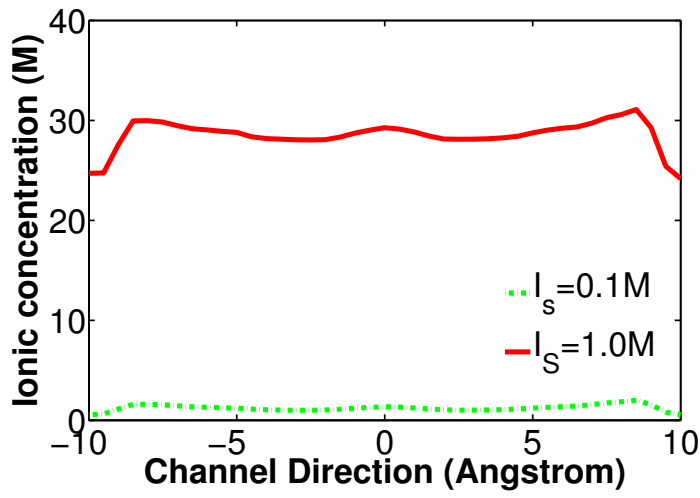

(c)

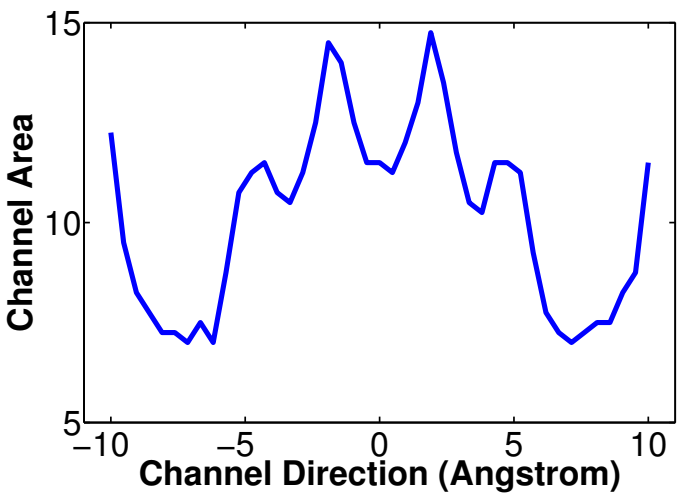

(b)

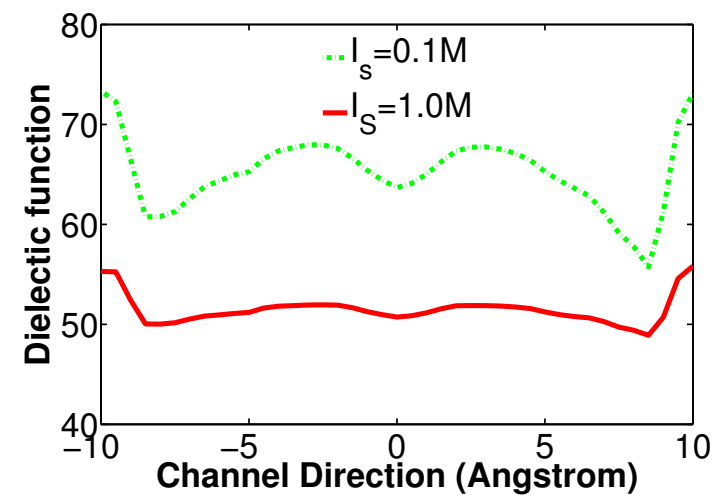

(d)

Figure 7: Simulated physical profiles of Gramicidin A channel by the QPBE $(\beta=12)$. (a) Electrostatic potential; (b) Estimated area of cross-section along channel direction; (c) Ionic concentration of cations; (d) dielectric property.

conclude that the simulations from the QPBE model suggest a stronger negative electrostatic environment than the PBE does for all the parameter combinations, and the difference can be as large as $2 k_{B} T / e$. Further, comparing Fig. 8 (a) with Fig. 8 (b), or Fig. 8 (c) with Fig. 8(d), we find out that for the same solvent (same value of $\beta$ ), the differences from the PBE and QPBE are less obvious if the ionic strength is stronger. Meanwhile, for the same ionic strength $I_{s}$, the differences are more significant when the ion species gives stronger dielectric decrement effect (larger value of $\beta$ ).

\section{Conclusions}

The traditional Poisson-Boltzmann equation is an effective implicit solvent model to study the ionic solvent or perform electrostatic analysis in molecular biosciences. It reduces the system complexity by treating water as a structureless continuum and by neglecting the impacts of mobile ions on the dielectric property of the solvent. Thus, this homogeneous assumption may lead to unphysical simulation results for ionic concentrations and other quantities near the strongly charged dielectric boundaries. One way to improve the accuracy and robustness of the mean-field PB theory is to model the solvent with a heterogeneous dielectric response. Experiments have shown that dielectric response of an ionic solvent decreases approximately linearly upon the ionic concentrations in the physiological condition, depending on various ionic species. Based on this observation, we proposed a mean-field model, in terms of a total free energy functional, to study electrostatics 

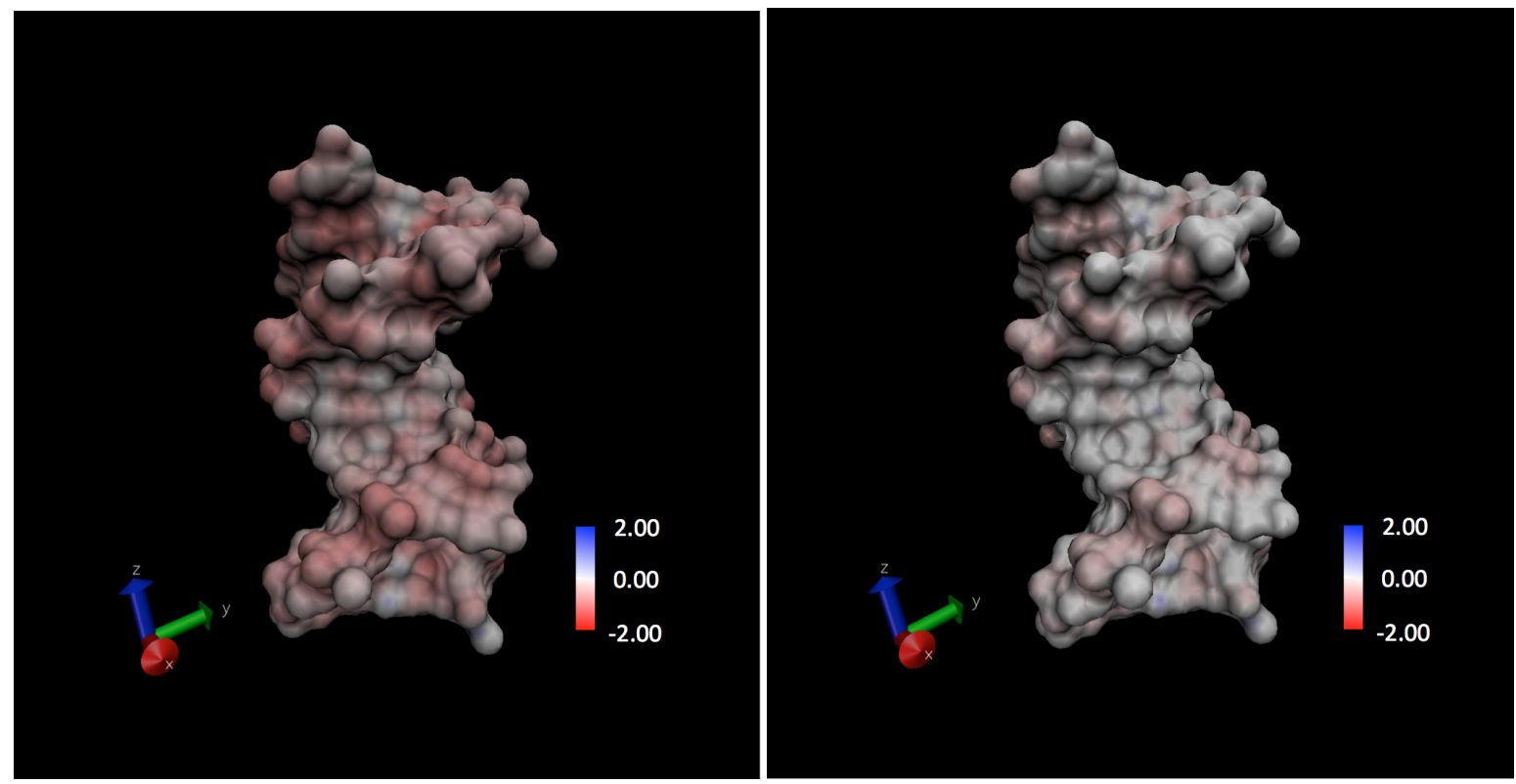

(a)

(b)
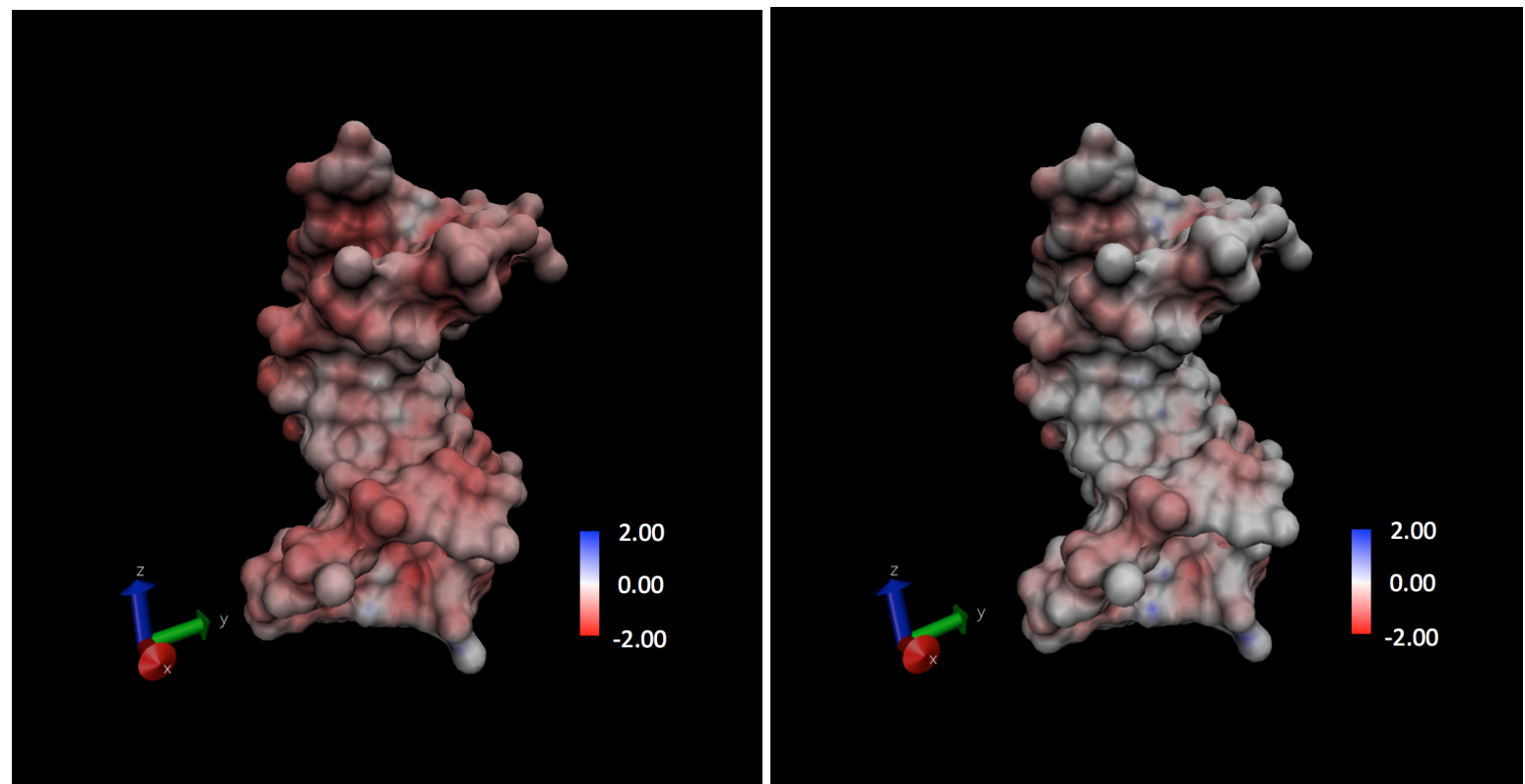

(c)

(d)

Figure 8: Differences of electrostatics (unit: $k_{B} T / e$ ) of a DNA segment (PDB code: 1D65) calculated by the QPBE and the PBE, are mapped to the molecular surface. (a): $I_{S}=0.2, \beta=12$; (b): $I_{S}=1.5, \beta=12$; (c) $I_{S}=0.2, \beta=20$; (d): $I_{S}=1.5, \beta=20$.

of biological molecules in a solvent with heterogeneous dielectric response. Based on experimental results, a linear dielectric function with respect to ionic concentration was considered in the energy functional. With this simple assumption, an interesting Poisson-Boltzmann type equation with an additional dielectric decrement parameter was derived for the system in equilibrium states.

The derived quasilinear Poisson-Boltzmann equation gives rise to many difficulties in finding its numerical solutions by iterative methods. A systematic way of finding the Jacobian matrix of the Newton's iterative method was developed in the current work. Besides this, an alternative iteration method, the fixed-point- 
Newton's method, was also developed. This iterative algorithms is associated with a much simpler Jacobian matrix but contains one more iterative loop. Numerical tests upon various parameter combinations indicated that both of the two algorithms can obtain the same convergent numerical solutions. Although numerous tests showed that the efficiency of the fixed-point-Newton's method is relatively lower and depends on the equation parameters, it is easier to perform and is a more general method for equations with discontinuous coefficients.

The proposed model and computational algorithms were applied to perform electrostatic analysis for biomacromolecules in an implicitly described solvent. In these applications, the QPBE was associated with a discontinuous dielectric function and singular charges for the source term due to the multiscale and multi domain system. Hence, a set of highly accurate interface algorithms were companied with the fixed-pointNewton's iteration to handle these challenges. Three examples in bimolecular sciences, including calculation of electrostatic solvation free energy, pore properties of ion channels, and surface electrostatics of a DNA strand, were presented as the QPBE simulations. It was concluded that the QPBE model can provide more physical details and fairly different electrostatic profiles near charged molecular surfaces. These new perspectives of this mean-field model are worthwhile to be justified by biological experiments. Mathematically, the well-posedness of the proposed nonlinear equation and the dependence of its solution on parameters remain interesting while open problems, which will be investigated in future work.

Acknowledgement: This work is funded by the Faculty Research Grant provided by the University of North Carolina at Charlotte.

\section{Appendix: Treatment of interface and singular charges in bimolecular applications}

To avoid singularities in the molecular surface, the interface $\Gamma$ is represented by the level-set function $S(\mathbf{r})=$ $\frac{1}{2}$, which is the solution of the steady state of the nonlinear parabolic equation

$$
\frac{\partial S}{\partial t}=|\nabla S| \nabla \cdot\left(\gamma \frac{\nabla S}{|\nabla S|}\right),
$$

with the boundary condition $S=0$ on $\partial \Omega \times[0, \infty)$ and the initial condition is a smooth function between 0 and 1 such that

$$
S(\mathbf{r}, 0)= \begin{cases}1, & \mathbf{r} \in \bigcup_{i=1}^{N_{a}}\left\{\mathbf{r}:\left|\mathbf{r}-\mathbf{r}_{i}\right|<r_{i}^{\mathrm{VWW}}+r_{p}\right\} \\ 0, & \text { otherwise }\end{cases}
$$

the parameters $r_{i}^{\mathrm{VDW}}$ and $r_{p}$ are the atomic van der Waals radius and the probe radius, respectively. Equation (A.1) is a simplified version of the differential geometry based molecular surface (see [37, 50, 51]), neglecting the ion solvent-interface interaction term.

Under this setup we have the following scaled interface problem of QPBE

$$
-\nabla \cdot(\epsilon(\mathbf{r}) \nabla u)+c_{2} I_{s} e^{-c_{1} \beta|\nabla u|^{2}} \sinh u=c_{3} \sum_{i}^{N_{a}} z_{i} \delta\left(\mathbf{r}-\mathbf{r}_{i}\right),
$$

where $\epsilon(\mathbf{r})$ is defined in Eq. (27). The parameters are the same as those in Eq. (9). The interface condition is

$$
\begin{aligned}
& {\left.[u]\right|_{\Gamma}=u^{+}(\mathbf{r})-u^{-}(\mathbf{r})=0,} \\
& {\left.[\epsilon \nabla u \cdot \overrightarrow{\mathbf{n}}]\right|_{\Gamma}=\epsilon^{+} \nabla u^{+} \cdot \overrightarrow{\mathbf{n}}-\epsilon^{-}(\mathbf{r}) \nabla u^{-} \cdot \overrightarrow{\mathbf{n}}=0}
\end{aligned}
$$


where superscripts "+” and "-” represent the limiting values of a certain function at two sides of interface $\Gamma$, and $\overrightarrow{\mathbf{n}}$ is the unit outward normal direction of $\Gamma$. Equations (A.4) and (A.5) guarantee the continuities of the potential function and its flux.

We adopt the technique in [17] to handle the singularity of the charge source, i.e., let

$$
u=\bar{u}+\tilde{u}
$$

where $\bar{u}$ and $\tilde{u}$ denote the singular part and regular part of $u$, respectively. More specifically, $\bar{u}$ should correspond to the singular delta function term and vanish outside $\Omega^{+}$, while $\tilde{u}$ is defined in the whole domain. By this consideration, we split $\bar{u}(\mathbf{r})$ as

$$
\bar{u}(\mathbf{r})=u^{\star}(\mathbf{r})+u^{0}(\mathbf{r})
$$

where

$$
u^{\star}(\mathbf{r})=c_{3} \sum_{i=1}^{N_{a}} \frac{z_{i}}{\epsilon^{+}\left|\mathbf{r}-\mathbf{r}_{i}\right|}
$$

represents the Coulomb's potential from the protein fixed charges. Since $\bar{u}(\mathbf{r})$ is required to vanish outside the $\Omega^{+}$as well as the boundary $\partial \Omega^{+}$, the $u^{\star}(\mathbf{r})$ should be corrected by $u^{0}(\mathbf{r})$, which is a harmonic function on $\Omega^{+}$ and

$$
u^{0}(\mathbf{r})=-u^{\star}(\mathbf{r}), \quad \forall \mathbf{r} \in \partial \Omega^{+}
$$

For the regular part $\tilde{u}$, we can take the advantage of the fact that $I_{s}$ is zero in $\Omega^{+}$, and the system (30)-(32) follows.

\section{References}

[1] B. Honig, A. Nicholls, Classical electrostatics in biology and chemistry, Science 268 (5214) (1995) 1144-9.

[2] R. Luo, L. David, M. K. Gilson, Accelerated Poisson-Boltzmann calculations for static and dynamic systems, Journal of Computational Chemistry 23 (13) (2002) 1244-53.

[3] J. Warwicker, H. C. Watson, Calculation of the electric potential in the active site cleft due to alpha-helix dipoles, Journal of Molecular Biology 157 (4) (1982) 671-9.

[4] W. Im, D. Beglov, B. Roux, Continuum solvation model: electrostatic forces from numerical solutions to the PoissonBoltzmann equation, Computer Physics Communications 111 (1-3) (1998) 59-75.

[5] N. A. Baker, D. Sept, S. Joseph, M. J. Holst, J. A. McCammon, Electrostatics of nanosystems: Application to microtubules and the ribosome, Proceedings of the National Academy of Sciences of the United States of America 98 (18) (2001) 10037-10041.

[6] A. H. Boschitsch, M. O. Fenley, Hybrid boundary element and finite difference method for solving the nonlinear PoissonBoltzmann equation, Journal of Computational Chemistry 25 (7) (2004) 935-955.

[7] R. J. Zauhar, R. S. Morgan, A new method for computing the macromolecular electric potential, Journal of Molecular Biology 186 (4) (1985) 815-20.

[8] I. Klapper, R. Hagstrom, R. Fine, K. Sharp, B. Honig, Focussing of electric fields in the active site of cu-zn superoxide dismutase: Effects of ionic strength and amino acid modification, Protein 1 (1986) $47-59$.

[9] N. V. Prabhu, M. Panda, Q. Y. Yang, K. A. Sharp, Explicit ion, implicit water solvation for molecular dynamics of nucleic acids and highly charged molecules, J. Comput. Chem. 29 (2008) 1113-1130.

[10] J. D. Madura, J. M. Briggs, R. C. Wade, M. E. Davis, B. A. Luty, A. Ilin, J. Antosiewicz, M. K. Gilson, B. Bagheri, L. R. Scott, J. A. McCammon, Electrostatics and diffusion of molecules in solution - simulations with the University of Houston Brownian Dynamics program, Computer Physics Communications 91 (1-3) (1995) 57-95.

[11] M. Engels, K. Gerwert, D. Bashford, Computational studies on bacteriorhodopsin: Conformation and proton transfer energetics, Biophys. Chem. 56 (1995) 95.

[12] M. Holst, N. Baker, F. Wang, Adaptive multilevel finite element solution of the Poisson-Boltzmann equation I. algorithms and examples, Journal of Computational Chemistry 21 (15) (2000) 1319-1342.

[13] B. Z. Lu, W. Z. Chen, C. X. Wang, X. J. Xu, Protein molecular dynamics with electrostatic force entirely determined by a single Poisson-Boltzmann calculation, Proteins 48 (3) (2002) 497-504.

[14] S. Jo, M. Vargyas, J. Vasko-Szedlar, B. Roux, W. Im, Pbeq-solver for online visualization of electrostatic potential of biomolecules, Nucleic Acids Research 36 (2008) W270-W275.

[15] B. R. Brooks, R. E. Bruccoleri, B. D. Olafson, D. States, S. Swaminathan, M. Karplus, Charmm: A program for macromolecular energy, minimization, and dynamics calculations, J. Comput. Chem. 4 (1983) 187-217. 
[16] Y. C. Zhou, M. Feig, G. W. Wei, Highly accurate biomolecular electrostatics in continuum dielectric environments, Journal of Computational Chemistry 29 (2008) 87-97.

[17] W. Geng, S. Yu, G. W. Wei, Treatment of charge singularities in implicit solvent models, Journal of Chemical Physics 127 (2007) 114106.

[18] D. Chen, Z. Chen, C. Chen, W. H. Geng, G. W. Wei, MIBPB: A software package for electrostatic analysis, J. Comput. Chem. 32 (2011) $756-770$.

[19] R. J. LeVeque, Z. L. Li, The immersed interface method for elliptic equations with discontinuous coefficients and singular sources, SIAM J. Numer. Anal. 31 (1994) 1019-1044.

[20] Z. L. Li, K. Ito, Maximum principle preserving schemes for interface problems with discontinuous coefficients, SIAM J. Sci. Comput. 23 (2001) 339-361.

[21] W. Geng, R. Krasny, A treecode-accelerated boundary integral Poisson-Boltzmann solver for continuum electrostatics of solvated biomolecules, Journal of Computational Physics 247 (20132-87) 6.

[22] A. Hildebrant, R. Blossey, S. Rjasanow, O. Kohlbacher, H. Lenhof, Novel Formulation of nonlocal electrostatics, Physical Review Letter 93 (2004) 108101-1.

[23] D. Ben-Yaakov, D. Andelman, R. Podgornik, R. Podgornik, lon-specific hydration effects: Extending the Poisson-Boltzmann theory, Current Opinion in Colloid and Interface Science 16 (2011) 542-550.

[24] I. Borukhov, D. Andelman, H. Orland, Adsorption of large ions from an electrolyte solution: a modified Poisson-Boltzmann equation, Electrochim Acta 46 (2000) 221-9.

[25] D. Ben-Yaakov, D. Andelman, R. Podgornik, Dielectric decrement as a source of ion-specific effects, J Chem. Phys. 134 (2011) 074705.

[26] M. Z. Bazant, B. D. Storey, A. A. Kornyshev, Double layer in ionic liquids: Overscreening versus crowding, Physical Review Letters 106 (2011) 046102.

[27] D. Gillespie, W. Nonner, R. S. Eisenberg, Density functional theory of charged, hard-sphere fluids, Phys Rev E 68 (2003) 1-10.

[28] B. Li, P. Liu, Z. Xu, S. Zhou, Ionic size effects: generalized Boltzmann distributions, counterion stratification, and modified Debye length , Nonlinearity (2013) 2899-2922.

[29] Y. Hyon, B. Eisenberg, C. Liu, A mathematical model for the hard sphere repulsion in ionic solutions, Commun. Math. Sci. 9 (2011) 459-475.

[30] L. Hu, G. W. Wei, Nonlinear poisson equation for heterogeneous media, Biophys. J.

[31] D. Xie, Y. Jiang, L. Scott, Efficient algorithms for solving a nonlocal dielectric model for protein in ionic solvent, SIAM Journal on Scientific Computing 38 (2013) B1267-1284.

[32] H. Li, B. Lu, An ionic concentration and size dependent dielectric permittivity Poisson-Boltzmann model for biomolecular solvation studies, J. Chem. Phys. 141 (2014) 024115.

[33] G. Wei, Multiscale multiphysics and multidomain models I: Basic theory, Journal of Theoretical and Computational Chemistry 12 (2013) 1341006.

[34] G. W. Wei, Differential geometry based multiscale models, Bulletin of Mathematical Biology 72 (2010) $1562-1622$.

[35] B. S. Eisenberg, Y. K. Hyon, C. Liu, Energy variational analysis of ions in water and channels: Field theory for primitive models of complex ionic fluids, Journal of Chemical Physics 133 (2010) 104104.

[36] D. Chen, G. W. Wei, Quantum dynamics in continuum for proton transport I: Basic formulation, Commun. Comput. Phys. 13 (2013) 285-324.

[37] D. Chen, Z. Chen, G. W. Wei, Quantum dynamics in continuum for proton transport II: Variational solvent-solute intersurface, International Journal for Numerical Methods in Biomedical Engineering 28 (2012) 25-51.

[38] D. Chen, G. W. Wei, Quantum dynamics in continuum for proton transport III: Generalized correlation, J Chem. Phys. 136 (2012) 134109.

[39] D. Chen, G. W. Wei, Modeling and simulation of electronic structure, material interface and random doping in nano-electronic devices, J. Comput. Phys. 229 (2010) 4431-4460.

[40] J. Che, J. Dzubiella, B. Li, J. A. McCammon, Electrostatic free energy and its variations in implicit solvent models, Journal of Physical Chemistry B 112 (10) (2008) 3058-69.

[41] B. Li, X. Cheng, Z. Zhang, Dielectric boundary force in molecular solvation with the Poisson-Boltzmann free energy: A shape derivative approach, SIAM J. Applied Math. 71 (2011) 2093-2111.

[42] Y. Z. Wei, S. Sridhar, Dielectric spectroscopy up to $20 \mathrm{GHz}$ of LiCl/ $\mathrm{H}_{2} \mathrm{O}$ solutions, J. Chem. Phys. 92 (1990) $923-928$.

[43] Y. Z. Wei, P. Chiang, S. Sridhar, Ion size effects on the dynamic and static dielectric properties of aqueous alkali solutions, J. Chem. Phys. 96 (1992) 4596.

[44] R. Buchner, G. T. Hefter, P. M. May, Dielectric Relaxation of Aqueous NaCl Solutions, J. Chem. Phys. A 103 (1999) 1-9.

[45] S. Senapati, A. Chandra, Surface charge induced modifications of the structure and dynamics of mixed dipolar liquids at solid-liquid interfaces: A molecular dynamics simulation study, J. Chem. Phys. 113 (2000) 8817-8826.

[46] M. J. Holst, F. Saied, Numerical solution of the nonlinear Poisson-Boltzmann equation: developing more robust and efficient methods, Journal of Computational Chemistry 16 (3) (1995) 337-64.

[47] S. Zhao, Operator splitting ADI schemes for pseudo-time coupled nonlinear solvation simulations, Journal of Computational Physics 257 (2014) 1000-1021. 
[48] M. K. Gilson, M. E. Davis, B. A. Luty, J. A. McCammon, Computation of electrostatic forces on solvated molecules using the Poisson-Boltzmann equation, Journal of Physical Chemistry 97 (14) (1993) 3591-3600.

[49] K. A. Sharp, B. Honig, Calculating total electrostatic energies with the nonlinear Poisson-Boltzmann equatlon, Journal of Physical Chemistry 94 (1990) 7684-7692.

[50] Z. Chen, N. A. Baker, G. W. Wei, Differential geometry based solvation models I: Eulerian formulation, J. Comput. Phys. 229 (2010) 8231-8258.

[51] Z. Chen, N. A. Baker, G. W. Wei, Differential geometry based solvation models II: Lagrangian formulation, J. Math. Biol. 63 (2011) 1139-1200. 